\title{
A MATRIX INEQUALITY
}

H. O. CORDES

In his paper Beiträge zur Störungstheorie der Spektralzerlegung [2] E. Heinz has proved the following

Theorem. Let $A$ in $\mathfrak{A}$ and $B$ in $\mathfrak{B}$ be two selfadjoint positive operators of an Hilbert space $\mathfrak{S}$ and let $Q$ in $\mathfrak{Q}$ be any arbitrary linear closed operator with the adjoint $Q^{*}$ defined in $\mathfrak{Q}^{*}$. Let $\mathfrak{Q} \subset \mathfrak{A}$ and $\mathfrak{Q}^{*} \subset \mathfrak{B}$ and let

$$
\begin{aligned}
\|Q u\| & \leqq\|A u\|, \quad \text { for every } u \in \mathfrak{Q}, \\
\left\|Q^{*} u\right\| & \leqq\|B u\|, \quad \text { for every } u \in \mathfrak{Q}^{*} .
\end{aligned}
$$

Statement:

$$
|(Q u, v)| \leqq\left\|A^{v} u\right\|\left\|B^{1-v} v\right\|, \quad \text { for every } u \in \mathfrak{A}, v \in \mathfrak{B},
$$

and for every $0 \leqq \nu \leqq 1$.

Although several other proofs and even generalizations of this remarkable and interesting estimate have been published by $T$. Kato [4], J. Dixmier [1] and E. Heinz [3], we are going to present here one more proof which shows the statement under a different aspect again and which perhaps has the advantage of using only very elementary arguments.

Our main tool will be the following very simple

Lemma. Let $T$ be a linear operator of an n-dimensional euclidean space and let $(u, v),(u, v)_{0}$ be any two positive definite inner products defined for $u, v$ of this space. Let

$$
\|u\|=\{(u, u)\}^{1 / 2}, \quad\|u\|_{0}=\left\{(u, u)_{0}\right\}^{1 / 2}
$$

and let

$$
\begin{aligned}
\|T\| & =\sup _{\|u\|=1}\|T u\|, \\
\|T\|_{0} & =\sup _{\|u\|_{0}=1}\|T u\|_{0} .
\end{aligned}
$$

Statement: If $T$ is hermitian symmetric with respect to $(u, v)_{0}$, then

$$
\|T\|_{0} \leqq\|T\| \text {. }
$$

Proof. It is well known that

Received by the editors April 27, 1959. 


$$
\|T\|_{0}=\underset{i=1, \ldots, n}{\operatorname{Max}}\left|\lambda_{i}\right|
$$

where $\lambda_{i}, i=1, \cdots, n$ are the $n$ proper values of $T$ :

$$
T \phi_{i}=\lambda_{i} \phi_{i}, \quad\left(\phi_{i}, \phi_{k}\right)_{0}=\delta_{i k}, \quad i, k=1, \cdots, n .
$$

But for each $\lambda_{i}$ we obtain

$$
\left\|T \phi_{i}\right\|=\left|\lambda_{i}\right|\left\|\phi_{i}\right\| \leqq\|T \mid\| \phi_{i} \| .
$$

Since by assumption both inner products are positive definite it follows that

$$
\left|\lambda_{i}\right| \leqq\|T\|, \quad i=1, \cdots, n .
$$

Hence

$$
\left\|T_{0}\right\|=\operatorname{Max}\left|\lambda_{i}\right| \leqq\|T\|
$$

follows immediately.

In order to use the above lemma for the proof of our theorem we first restrict ourselves to the case of a finite dimensional Hilbert space $\mathfrak{S}$ and also we impose the further assumption that $A$ and $B$ both have a bounded inverse. These additional assumptions will be removed later.

By substitutions of the form $A u=v$, resp. $B u=v$ the two inequalities

$$
\|Q u\| \leqq\|A u\|, \quad\left\|Q^{*} u\right\| \leqq\|B u\|
$$

go into

$$
\left\|Q A^{-1} v\right\| \leqq\|v\|, \quad\left\|Q^{*} B^{-1} v\right\| \leqq\|v\|,
$$

Since the adjoint of every bounded operator is bounded by the same constant as the original operator, it follows that

$$
\left\|A^{-1} Q^{*} u\right\| \leqq\|u\|, \quad\left\|B^{-1} Q u\right\| \leqq\|u\| .
$$

Consequently

$$
\left\|A^{-1} Q^{*} B^{-1} Q\right\| \leqq 1 .
$$

Since the operator $T=A^{-1} Q^{*} B^{-1} Q$ is hermitian with respect to the positive definite inner product

$$
(u, v)_{0}=(u, A v),
$$

we get

$$
\|T\|_{0} \leqq\|T\| \leqq 1 .
$$

Now 


$$
(u, T u)_{0} \leqq(u, u)_{0}
$$

follows. Introducing again the inner product $(u, v)$ we get

$$
\left(Q u, B^{-1} Q u\right) \leqq(u, A u)
$$

or

$$
\left\|B^{-1 / 2} Q A^{-1 / 2} v\right\|^{2} \leqq\|v\|^{2} .
$$

Finally

$$
\left|\left(B^{-1 / 2} Q A^{-1 / 2} u, v\right)\right| \leqq\|u\|\|v\|
$$

or

$$
|(Q u, v)| \leqq\left\|A^{1 / 2} u\right\|\left\|B^{1 / 2} v\right\|
$$

follows.

This is the statement for $\nu=1 / 2$.

In order to prove the theorem for the general case we use an argument which looks similar to that used by $\mathrm{T}$. Kato [4], but it does not seem to be the same.

We assume the statement to be true for all $\nu=m / 2^{k}, m=0,1,2$, $\cdots, 2^{k}$ and we show that from there it follows by induction for every $\nu=m / 2^{k+1}, m=0,1,2, \cdots, 2^{k+1}$.

Since by the above arguments the statement has been shown to be true for $\nu=0,1 / 2,1$, that means for $k=1$, this amounts to a proof for every number of the type $k / 2^{m}, k, m$ being arbitrary.

From

$$
|(Q u, v)| \leqq\left\|A^{m / 2^{k}} u\right\|\left\|B^{1-m / 2^{k}} v\right\|
$$

we conclude that

$$
\left|\left(Q A^{-m / 2^{k}} u, v\right)\right| \leqq\|u\|\left\|u B^{1-m / 2^{k}} v\right\| .
$$

This amounts to

$$
\left\|A^{-m / 2^{k}} Q v\right\| \leqq\left\|B^{1-m / 2^{k}} v\right\| .
$$

On the other hand

$$
\left\|Q A^{-m / 2^{k}} u\right\| \leqq\left\|A^{1-m / 2^{k}} u\right\|
$$

follows from our initial assumption

$$
\|Q u\| \leqq\|A u\| \text {. }
$$

Now let

$$
Q_{1}=Q A^{-m / 2^{k}}, \quad A_{1}=A^{1-m / 2^{k}}, \quad B_{1}=B^{1-m / 2^{k}} .
$$


Then the above inequalities amount to

$$
\left\|Q_{1} u\right\| \leqq\left\|A_{1} u\right\|, \quad\left\|Q_{1}^{*} u\right\| \leqq\left\|B_{1} u\right\| .
$$

By application of the theorem for $\nu=1 / 2$ we get

$$
\left|\left(Q A^{-m / 2^{k}} u, v\right)\right| \leqq\left\|A^{1 / 2\left(1-m / 2^{k}\right)} u\right\|\left\|B^{1 / 2\left(1-m / 2^{k}\right)} v\right\|,
$$

or

$$
\begin{aligned}
& |(Q u, v)| \leqq\left\|A^{1 / 2+m / 2^{k+1}} u\right\|\left\|B^{1 / 2-m / 2^{k+1}} v\right\| \\
& |(Q u, v)| \leqq\left\|A^{\left(2^{k}+m\right) / 2^{k+1}} u\right\|\left\|B^{1-\left(2^{k}+m\right) / 2^{k+1}} v\right\| .
\end{aligned}
$$

Hence the assertion follows for

$$
\nu=\frac{2^{k}+m}{2^{k+1}}, \quad m=0,1,2, \cdots, 2^{k}
$$

and by reasons of symmetry for

$$
\nu=\frac{2^{k}-m}{2^{k+1}}
$$

$m=0,1,2, \cdots, 2^{k}$ too.

Together we conclude the assertion for all

$$
\nu=\frac{m}{2^{k+1}}, \quad m=0,1, \cdots, 2^{k+1} .
$$

Since finally $A^{\nu}$ and $B^{1-\nu}$ depend continuously on $\nu$ and since the set of all numbers of the form $m / 2^{k}$ is dense in the interval $0 \leqq \nu \leqq 1$ we get the assertion for every $\nu$ of this interval.

From the continuity of $A^{\nu}$ and $B^{1-\nu}$ in $A$ and $B$ follows further that the assumption of existence of $A^{-1}$ and $B^{-1}$ can be removed. For if $A^{-1} B^{-1}$ does not exist, then replace $A$ and $B$ by $A+\epsilon, B+\epsilon$, $\epsilon>0$, respectively. Because of

$$
\|A u\| \leqq\|(A+\epsilon) u\| \text { and }\|B u\| \leqq\|(B+\epsilon) u\|
$$

( $A$ and $B$ are positive definite) we conclude

$$
\|Q u\| \leqq\|(A+\epsilon) u\|, \quad\left\|Q^{*} u\right\| \leqq\|(B+\epsilon) u\| .
$$

Now $A+\epsilon, B+\epsilon$ have an inverse, hence

$$
|(Q u, v)| \leqq\left\|(A+\epsilon)^{v} u\right\|\left\|(B+\epsilon)^{1-v} v\right\|
$$

for every $\epsilon$. If $\epsilon$ tends to zero it follows

$$
|(Q u, v)| \leqq\left\|A^{v} u\right\|\left\|B^{1-\nu} v\right\|
$$


for the more general class of $A, B$ too.

Finally every conclusion except the proof of the lemma works for an infinitely dimensional Hilbert space too provided that all operators are assumed to be bounded. The lemma can easily be proved for this more general case too under the additional assumption that the two metrics $\|u\|$ and $\|u\|_{0}$ be topologically equivalent:

$$
c\|u\|_{0} \leqq\|u\| \leqq C\|u\|_{0} .
$$

The proof can be given as follows:

If $\|u\|_{0}$ and $\|u\|$ are equivalent norms then an operator is bounded in both norms if it is bounded in one of the two norms only. Hence the spectrum $\sigma$ of an operator $T$ (i.e. the set of $\lambda$ for which $(T-\lambda)^{-1}$ is bounded) is the same under both norms.

Let

$$
\left\|T\left|\|=\sup _{\lambda \in \sigma}\right| \lambda \mid\right.
$$

be the spectral norm of $T$.

Then, as is well known,

$$
\|T\|=\|T\|_{0}
$$

if $T$ is hermitian symmetric (or even only normal) under $(u, v)_{0}$ and

$$
\|T\|\|\| T \| \text {. }
$$

Hence

$$
\|T\|_{0} \leqq\|T\|
$$

and the lemma is proven.

Finally the extension of the theorem to the case of general unbounded operators follows in the same manner as in [2].

\section{REFERENCES}

1. J. Dixmier, Sur une inégalité de E. Heinz, Math. Ann. vol. 126 (1953) pp. 75-78.

2. E. Heinz, Beiträge zur Störungstheorie der Spektralzerlegung, Math. Ann. vol. 123 (1951) pp. 415-438.

3. - On an inequality for linear operators in a Hilbert space, Report of an International Conference on Operator Theory and Group Representations, Arden House, Harriman, N. Y., 1955, pp. 27-29. Publ. 387, National Academy of Sciences, National Research Council, Washington, D. C., 1955.

4. T. Kato, Notes on some inequalities for linear operators, Math. Ann. vol. 125 (1952) pp. 208-212.

University of California, Berkeley 